\title{
Site of hospital readmission and mortality: a population-based retrospective cohort study
}

\author{
John A. Staples MD MPH, Deva Thiruchelvam MSc, Donald A. Redelmeier MD MS(HSR)
}

\section{Abstract}

Background: Unplanned hospital readmission is a complex process, particularly if the patient is readmitted to an acute care institution other than the original hospital. This study tested the hypothesis that readmission to an alternative hospital is associated with increased mortality compared with readmission to the original hospital.

Methods: We performed a population-based retrospective cohort analysis set between 1995 and 2010 for all 21 acute care adult general hospitals in the Greater Toronto and Hamilton Area. Participants were consecutive adults (age $\geq 18 \mathrm{yr}$ ) readmitted through the emergency department within 30 days after hospital discharge. The primary outcome measure was all-cause mortality within 30 days after readmission.

Results: Of the 198149 patients included in the study, 38134 (19.2\%) died within 30 days after readmission. Patients readmitted to an alternative hospital were more likely than those readmitted to the original hospital to be older, reside in a chronic-care facility and arrive by ambulance. Alternative-hospital readmission was associated with a higher risk of death within 30 days (22.3\% v. $18.6 \%$, $p<0.001$; odds ratio [OR] 1.26, 95\% confidence interval [Cl] 1.23-1.30). The increased risk was substantially less after adjustment for patient- and hospital-level covariables (adjusted OR 1.06, 95\% Cl 1.02-1.10). Unadjusted Kaplan-Meier survival curves separated early and the absolute difference in mortality continued throughout the entire 1-year follow-up period, but no difference between groups was observed based on adjusted survival analyses.

Interpretation: Among patients readmitted within 30 days after discharge, readmission to an alternative hospital was associated with a higher risk of death than readmission to the original hospital. Whether this adverse prognosis reflects a true causal relation or residual confounding is unknown.

$\mathrm{E}$ ach year, about 4.5 million people in the United States and about 0.2 million Canadians undergo unplanned readmission to hospital within 30 days after hospital discharge. ${ }^{1,2}$ Unplanned readmissions are costly, challenging to predict, difficult to avoid and associated with increased mortality. ${ }^{3-9}$ Although most readmitted patients return to the original hospital, about a fifth spend their initial ("primary") and subsequent ("secondary") stays in different hospitals. ${ }^{10}$ Clinical experience suggests that unfamiliar patients can pose challenges, but 1 study found no large difference in mortality between patients readmitted to original and alternative hospitals. ${ }^{11}$

Patients readmitted to an alternative hospital might be disadvantaged by limited access to their primary admission medical records, delayed treatment, discontinuity of care and increased exposure to nosocomial pathogens. ${ }^{12-18}$ On the other hand, clinicians in an alternative hospital may be less likely to replicate errors committed during the primary admission, less influenced by previously affixed diagnostic labels and less dispirited by a patient's return to hospital. ${ }^{19}$ In addition, procedures and protocols at the alternative hospital may intercept problems arising in the original hospital and facilitate rectification of prior medical errors. ${ }^{20}$ These multiple countervailing forces have an uncertain aggregate influence on patient outcomes.

Whether a patient is readmitted to the original hospital or an alternative is determined by factors that include hospital proximity, specialist availability, ambulance referral patterns, local traffic conditions, anticipated emergency department delays, institutional reputation and patient preference..$^{21-24}$

Competing interests: None declared.

This article has been peer reviewed.

Correspondence to: John Staples, john.a.staples@gmail.com CMAJ Open 2014.DOI:10.9778/cmajo.20130053 
The complexity and heterogeneity of these factors make a prospective randomized trial logistically challenging and ethically dubious. Thus, we conducted a population-based retrospective cohort analysis using linked administrative databases to test whether readmission to an alternative hospital is associated with a higher risk of death than readmission to the original hospital.

\section{Methods}

\section{Setting}

We focused on a large metropolitan area, reasoning that readmissions to alternative hospitals in rural regions would be uncommon, subject to practicalities, such as distances, and confounded by referrals for urban subspecialty care. We selected Ontario's Greater Toronto and Hamilton Area because it is Canada's largest contiguous urban region. ${ }^{25}$ At the midpoint of our study (2002), the area covered an area of 8241 $\mathrm{km}^{2}$ and had a population of 5.6 million. ${ }^{26}$ Throughout the study interval, Ontario residents had universal health insurance that provided access to medical care that was widely available, publicly funded and free at the point of service. ${ }^{27}$ The health care system within the Greater Toronto and Hamilton Area included a full array of primary, secondary, tertiary and quaternary care, and no major changes to hospital financing occurred during the study.

\section{Hospitals}

We performed an individual-level analysis of patients whose primary and secondary admissions to hospital both occurred within the study area. This approach minimized confounding by excluding patients referred from rural regions for subspecialty care. We first identified all acute care hospitals operating in the study area between 1995 and 2010. Individual inpatient acute care sites sharing the same facility identification number were classified as a multisite hospital because their shared data information systems, personnel, protocols and governance made that the smallest meaningful functional unit for analysis. Individual inpatient acute care sites with no sister sites were classified as single-site hospitals. We excluded hospitals without adult inpatient acute care beds and hospitals restricted to pediatric, elective surgical, rehabilitative, respite or palliative care. Inpatient acute care sites that joined a multisite hospital during the study period were excluded before the merger date.

\section{Patients}

We identified adult patients aged 18 years or older who were associated with 1 or more eligible hospital readmissions during the study interval. A readmission was eligible if the patient was readmitted to hospital through the emergency department between Jan. 1, 1995, and Dec. 31, 2010; the time between initial hospital discharge and subsequent hospital readmission was 1-30 days; and both the primary and secondary admissions occurred in qualifying hospitals in the study area. A readmission was ineligible if the most responsible diagnosis at the primary or secondary admission was related to pregnancy (International Classification of Diseases, 9th Revision [ICD-9] codes 630-676; International Statistical Classification of Diseases and Related Health Problems, Tenth Revision, Canada [ICD-10-CA] codes O00-O99) or a major psychiatric disorder (ICD-9 codes 295-300; ICD-10CA codes F20-F48). Same-day readmissions were excluded to avoid misidentifying hospital-to-hospital transfers as readmissions. For patients with multiple readmissions, only the most recent eligible readmission was analyzed, so that each individual contributed only once to analyses. (See eFigure 1 in Appendix 1 available at www.cmajopen.ca/content/2/2/E77 /suppl/DC1 for a schematic representation of the study.)

The Research Ethics Board of the Sunnybrook Research Institute approved the study and waived the requirement for individual consent.

\section{Variables}

The primary outcome was all-cause mortality within 30 days after hospital readmission, as ascertained from the official government vital statistics record for Ontario. Secondary outcomes were in-hospital case fatality and all-cause mortality by 90, 180 and 365 days after readmission. The predictor of interest was the site of readmission, categorized as original hospital or alternative hospital. Original-hospital readmissions were defined as those where both primary and secondary admissions occurred at the same hospital. Alternative-hospital readmissions were those where primary and secondary admissions occurred at different hospitals.

Covariables used for adjusted analyses were selected based on earlier research and included patient demographics, neighbourhood average household income (divided into quintiles), rural residence (yes, no), year of readmission, Charlson Comorbidity Index (integer), primary length of stay (days), most responsible diagnosis for secondary hospital admission, arrival by ambulance at the time of readmission (yes, no), residence in a chronic care facility at time of readmission (yes, no), total number of admissions to hospital in the year before readmission (integer) and total number of physician clinic visits in the year before readmission (integer). ${ }^{5,28}$ We included the hospital-free interval, defined as the number of days between hospital discharge and readmission. On the basis of earlier reports, we also examined annual case volume (integer) and sector (designated as academic sector or community sector by system-level hospital reports) of the readmission hospital. ${ }^{29-33}$ Variables were modelled as continuous values unless otherwise indicated but are presented in categories to facilitate interpretation.

In the Canadian setting, the condition most responsible for a patient's hospital stay is reported as the most responsible diagnosis. ${ }^{34}$ In our study, the most responsible diagnosis for each admission was coded according to the ICD-9 before Apr. 1, 2002, and the ICD-10-CA after Apr. 1, 2002. We adapted the 285 single-level diagnostic categories in the Agency for Healthcare Research and Quality's Clinical Classifications Software to cluster individual diagnostic codes into 30 mutually exclusive, clinically meaningful diagnostic categories (eTable 1 in Appendix 1). ${ }^{35}$ 


\section{Data sources}

Encrypted patient-level data were obtained from populationbased, linked administrative databases that have been used extensively in research ${ }^{17,36,37}$ and validated. ${ }^{38-41}$ Information on outpatient clinic visits was obtained from the Ontario Health Insurance Plan database. Information on hospital admissions was obtained from the Canadian Institute for Health Information database. Demographics, vital status and date of death were obtained from official government records. Socioeconomic status was estimated using average household income at the census tract level based on the 2006 Canadian census. Hospital structural and operational data (including designation as an academic or community hospital) were obtained from reports published at or near the study midpoint. ${ }^{29,42-44}$ The study interval extended from 1995 to 2010 to include the most recent year for which data were available and to reflect an interval with no major changes in hospital financing.

Missing data for neighbourhood average household income and rural residence were coded explicitly as missing and retained for analysis (less than $0.1 \%$ of patients). Patientlevel data were otherwise complete.

\section{Statistical analysis}

The primary analysis compared 30-day mortality after alternative-hospital readmission with 30-day mortality after original-hospital readmission. All analyses were at the level of the individual patient. We compared unadjusted risks using a $\chi^{2}$ test and then adjusted for all patient- and hospital-level covariables listed above using a logistic regression model that applied general estimating equations (GEE) with an exchangeable correlation structure to account for clustering of individuals within hospitals. ${ }^{45}$ In a subsequent patient-level analysis, we stratified patients by secondary hospital, calculated unadjusted odds ratios (ORs) for each secondary hospital stratum, and then calculated adjusted ORs for each secondary hospital stratum by fitting separate logistic regression models to each hospital to account for the individual patient characteristics listed above. In further analyses, we stratified the pooled cohort by patient characteristics and used unadjusted univariable GEE to explore potential effect modification.

Kaplan-Meier survival curves were generated for unadjusted survival up to 1 year and tested using the log-rank test. Survival analysis was done using a Cox proportional-hazards analysis adjusted for all patient- and hospital-level characteristics listed above. In-hospital case fatality and death by 90, 180 and 365 days were examined using the multivariable GEE logistic regression model developed for the primary outcome. One sensitivity analysis retained only the first (rather than most recent) eligible readmission for each patient. A second sensitivity analysis examined the influence of analyzing according to primary (rather than secondary) hospital. A third sensitivity analysis defined all patients readmitted within 48 hours as hospital-tohospital transfers and excluded those patients from analysis. A post hoc exploratory analysis examined the relative influence of distinct hospital-sector transitions (Appendix 1). We performed all analyses using 2 -sided statistical tests at the $5 \%$ level of significance using SAS, version 9.2 (SAS Institute Inc., Cary, NC).

\section{Results}

\section{Hospital and patient characteristics}

A total of 43 inpatient acute care sites grouped within 28 distinct hospitals were active in the Greater Toronto and Hamilton Area during the study period. Seven single-site hospitals provided highly restricted subspecialty care and were excluded from analysis ( 3 provided only elective surgical services, 3 provided only palliative care and 1 provided only pediatric care), leaving 21 qualifying acute care hospitals (eFigure 2 in Appendix 1). Hospital characteristics at the midpoint of the study can be found in eTables 2 and 3 in Appendix 1.

We identified 2448759 patients who were admitted at least once to a qualifying acute care hospital in the study area during the 16-year study period; of these, 198228 (8.1\%) subsequently had an eligible readmission to a hospital in the study area (Figure 1). We excluded 79 patients, whose date of death was erroneously recorded as occurring before the readmission date, resulting in a cohort of 198149 patients. Of these, $58460(29.5 \%)$ patients had more than 1 eligible readmission (eFigure 3 in Appendix 1), but only the most recent readmission episode for each patient was retained in the primary analysis. Less than $0.003 \%$ of data were missing for age, sex, neighbourhood average household income, prior physician visits, prior admissions to hospital and most responsible diagnosis at primary and secondary admission.

Of the 198149 patients in our cohort, 161974 (81.7\%) were readmitted to the original hospital and 36175 (18.3\%) to an alternative hospital. Compared with patients readmitted to the original hospital, those readmitted to an alternative hospital were more likely to be older, male, burdened by more comorbidity (as measured by hospital admissions and clinic visits in the previous year and by Charlson Comorbidity Index), a resident of a chronic care facility and brought to hospital by

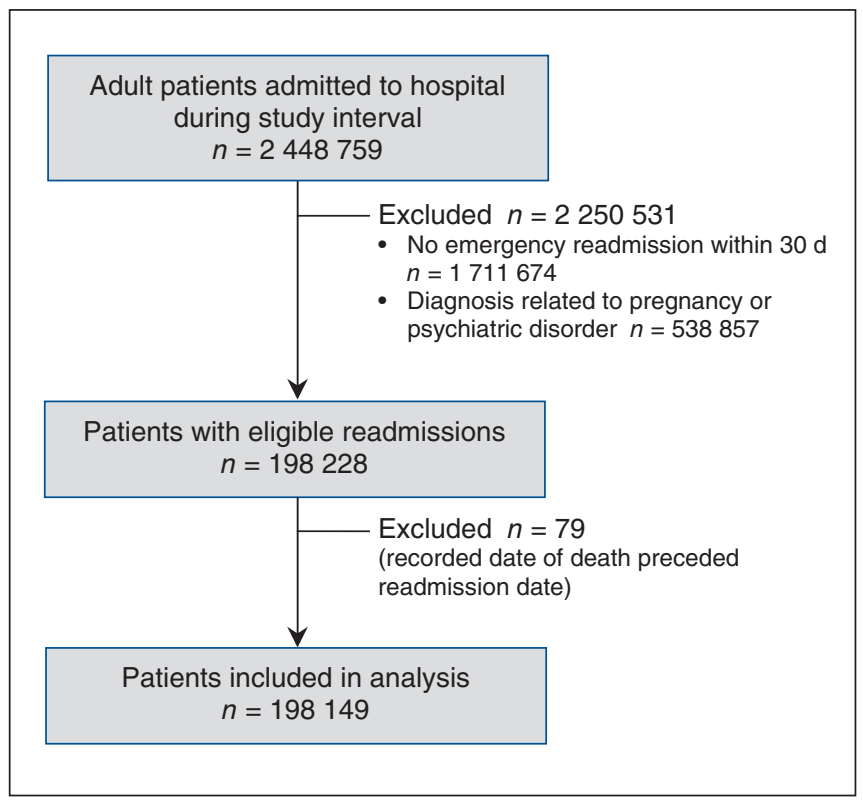

Figure 1: Selection of patients readmitted to hospital in the Greater Toronto and Hamilton Area within 30 days after discharge. 


\section{OPEN}

Research

ambulance (Table 1). Compared with original-hospital readmissions, alternative-hospital readmissions were somewhat more likely to occur at community hospitals or at those with a lower annual case volume (e'Table 6 in Appendix 1).

\section{Short-term mortality}

Compared with patients readmitted to the original hospital, those readmitted to an alternative hospital were significantly more likely to die within 30 days after readmission $(8072$ [22.3\%] of 36175 patients v. 30062 [18.6\%] of 161974 patients; $\chi^{2}$ test, $\left.p<0.001\right)$. This difference is equivalent to an unadjusted OR of 1.26 (95\% confidence interval [CI] 1.23 1.30). Adjustment for patient and hospital factors substantially attenuated this association (adjusted OR 1.06, 95\% CI 1.021.10). Additional independent predictors of death appear in Table 2. In our final model, the relative increase in risk of death within 30 days attributable to alternative-hospital readmission was generally smaller than the risk associated with sepsis or pneumonia (Table 2). The within-hospital correlation coefficient from our primary analysis was 0.12 .

Alternative-hospital readmission was associated with a significant increase in the adjusted odds of death within 30 days

Table 1: Characteristics of patients readmitted to the original hospital or an alternative hospital

\begin{tabular}{|c|c|c|c|}
\hline \multirow[b]{2}{*}{ Characteristic } & \multicolumn{2}{|c|}{ Readmission hospital; no. (\%) of patients } & \multirow[b]{2}{*}{$p$ value } \\
\hline & $\begin{array}{c}\text { Original hospital } \\
n=161974\end{array}$ & $\begin{array}{l}\text { Alternative hospital } \\
\qquad n=36175\end{array}$ & \\
\hline \multicolumn{4}{|l|}{ Age, $y r^{*}$} \\
\hline$<65$ & $63578(39.3)$ & $13064(36.1)$ & $<0.001$ \\
\hline $65-74$ & $33471(20.7)$ & $7602(21.0)$ & $<0.001$ \\
\hline $75-84$ & $41941(25.9)$ & $9650(26.7)$ & $<0.001$ \\
\hline$\geq 85$ & $22984(14.2)$ & $5859(16.2)$ & $<0.001$ \\
\hline Sex, female & $80941(50.0)$ & $17654(48.8)$ & $<0.001$ \\
\hline \multicolumn{4}{|l|}{ Neighbourhood average household income } \\
\hline Highest quintile & $29375(18.1)$ & $6675(18.5)$ & $<0.001$ \\
\hline Next to highest quintile & $29277(18.1)$ & $6381(17.6)$ & $<0.001$ \\
\hline Middle quintile & $30910(19.1)$ & 6609 (18.3) & $<0.001$ \\
\hline Next to lowest quintile & $35262(21.8)$ & $7653(21.2)$ & $<0.001$ \\
\hline Lowest quintile & $36696(22.7)$ & $8732(24.1)$ & $<0.001$ \\
\hline Rural residence† & $3198 \quad(2.0)$ & $465 \quad(1.3)$ & $<0.001$ \\
\hline Additional admissions to hospital in previous year* & $81549(50.3)$ & $19428(53.7)$ & $<0.001$ \\
\hline$\geq 7$ physician clinic visits in previous year* & $125726(77.6)$ & $28540(78.9)$ & $<0.001$ \\
\hline Charlson Comorbidity Index $\geq 2^{*}$ & $84955(52.4)$ & $19704(54.5)$ & $<0.001$ \\
\hline \multicolumn{4}{|l|}{ Most responsible diagnosis for primary admission $\ddagger$} \\
\hline Malignant neoplasm & $19590(12.1)$ & $4303(11.9)$ & 0.3 \\
\hline Injury & $10666 \quad(6.6)$ & $2724 \quad(7.5)$ & $<0.001$ \\
\hline Acute coronary syndrome & $9000 \quad(5.6)$ & $2115 \quad(5.8)$ & 0.030 \\
\hline Heart failure & $9258 \quad(5.7)$ & $1856 \quad(5.1)$ & $<0.001$ \\
\hline Pneumonia & $6696 \quad(4.1)$ & $1588 \quad(4.4)$ & 0.03 \\
\hline Obstructive lung disease & $6742 \quad(4.2)$ & $1316 \quad(3.6)$ & $<0.001$ \\
\hline Chronic ischemic heart disease & $3168 \quad(2.0)$ & $2440 \quad(6.7)$ & $<0.001$ \\
\hline Cardiac arrhythmia & $4318 \quad(2.7)$ & $824 \quad(2.3)$ & $<0.001$ \\
\hline Cerebrovascular disease & $3708 \quad(2.3)$ & $1135 \quad(3.1)$ & $<0.001$ \\
\hline Miscellaneous & $49237(30.4)$ & $10652(29.4)$ & $<0.001$ \\
\hline Primary length of stay* greater than cohort median & $92533(57.1)$ & $20843(57.6)$ & 0.09 \\
\hline Hospital-free interval $^{\star}$ greater than cohort median & $80749(49.9)$ & $20321(56.2)$ & $<0.001$ \\
\hline Residence in chronic care facility at readmission & $16479(10.2)$ & $5446(15.1)$ & $<0.001$ \\
\hline Arrival by ambulance at readmission & $70523(43.5)$ & 20677 (57.2) & $<0.001$ \\
\hline
\end{tabular}


for 4 of 6 academic secondary hospitals. For all other secondary hospitals, there was no significant difference in the adjusted risk of death between original-hospital and alternative-hospital readmissions (Figure 2; e'Table 7 in Appendix 1). Post hoc stratification of the multivariable GEE model by sector of the secondary hospital showed a significant association between 30-day mortality and alternative-hospital readmission for academic hospitals (adjusted OR 1.17, 95\% CI 1.15-1.19), but not for community hospitals (adjusted OR $1.00,95 \%$ CI $0.97-1.04)$. Results of an exploratory analysis examining specific hospital sector transitions yielded similar findings (e'Table 9 in Appendix 1).

The unadjusted association between alternative-hospital readmission and increased mortality was present among patients of all age groups, all neighbourhood household income quintiles and both sexes (Table 3). The unadjusted relative increase in risk was accentuated among women and among patients with less comorbidity, fewer admissions to hospital and a residence other than a chronic care facility.

\section{Delayed mortality}

Unadjusted survival analysis showed that the risk associated with alternative-hospital readmission was equal to an absolute difference in mortality of about $4 \%$ that was sustained throughout the 1-year follow-up period (Figure 3; log rank test $p<0.001)$. In contrast, adjusted survival analysis found no difference between the groups (hazard ratio $1.01,95 \% \mathrm{CI}$ 0.99-1.02). Adjusted analyses at specific times revealed that

Table 2: Factors associated with death within $\mathbf{3 0}$ days after hospital readmission

\begin{tabular}{|c|c|c|c|}
\hline Factor & $\begin{array}{l}\text { Adjusted } \mathrm{OR}^{*} \\
\quad(95 \% \mathrm{Cl})\end{array}$ & Factor & $\begin{array}{l}\text { Adjusted OR* } \\
\quad(95 \% \mathrm{Cl})\end{array}$ \\
\hline \multicolumn{2}{|l|}{ Age, yr† } & \multirow{2}{*}{\multicolumn{2}{|c|}{$\begin{array}{l}\text { Residence in chronic care facility } \\
\text { at readmission }\end{array}$}} \\
\hline$<65$ (ref) & 1.00 & & \\
\hline $65-74$ & $1.26(1.23-1.30)$ & No (ref) & 1.00 \\
\hline $75-84$ & $1.49(1.43-1.55)$ & Yes & $1.31(1.25-1.37)$ \\
\hline$\geq 85$ & $2.05(1.90-2.21)$ & \multirow{2}{*}{\multicolumn{2}{|c|}{$\begin{array}{l}\text { Most responsible diagnosis } \\
\text { for readmission§ }\end{array}$}} \\
\hline \multicolumn{2}{|l|}{ Sex } & & \\
\hline Female (ref) & 1.00 & Cardiac arrest & $3.00(5.70-9.18)$ \\
\hline Male & $1.15(1.13-1.16)$ & Malignant neoplasm & $3.57(3.16-4.03)$ \\
\hline \multicolumn{2}{|l|}{ Neighbourhood household incomeł } & Sepsis & $3.10(2.72-3.53)$ \\
\hline Highest quintile (ref) & 1.00 & Liver disease & $2.29(2.03-2.59)$ \\
\hline Next to highest quintile & $0.99(0.96-1.03)$ & Pneumonia & $1.87(1.69-2.07)$ \\
\hline Middle quintile & $1.01(0.98-1.04)$ & Nephrolithiasis & $0.44(0.41-0.47)$ \\
\hline Next to lowest quintile & $1.03(0.98-1.09)$ & Nonspecific abdominal pain & $0.40(0.36-0.43)$ \\
\hline Lowest quintile & $1.01(0.98-1.05)$ & Nonspecific chest pain & $0.26(0.24-0.28)$ \\
\hline \multirow{2}{*}{\multicolumn{2}{|c|}{$\begin{array}{l}\text { Admissions to hospital in } \\
\text { previous year† }\end{array}$}} & Syncope and collapse & $0.24(0.22-0.27)$ \\
\hline & & Prostatic hypertrophy & $0.24(0.20-0.29)$ \\
\hline 1 (ref) & 1.00 & Miscellaneous (ref) & 1.00 \\
\hline$\geq 2$ & $1.11(1.09-1.14)$ & \multicolumn{2}{|l|}{ Arrival by ambulance at readmission } \\
\hline \multirow{2}{*}{\multicolumn{2}{|c|}{$\begin{array}{l}\text { Physician clinic visits in } \\
\text { previous year } †\end{array}$}} & No (ref) & 1.00 \\
\hline & & Yes & $1.95(1.88-2.01)$ \\
\hline$\leq 6$ (ref) & 1.00 & \multicolumn{2}{|l|}{ Year of readmission $†$} \\
\hline$\geq 7$ & $0.97(0.94-1.00)$ & 1994-1999 (ref) & 1.00 \\
\hline \multicolumn{2}{|l|}{ Charlson Comorbidity Index $†$} & 2000-2005 & $0.95(0.92-0.99)$ \\
\hline$\leq 1$ (ref) & 1.00 & 2006-2010 & $0.87(0.83-0.92)$ \\
\hline$\geq 2$ & $1.87(1.80-1.94)$ & \multicolumn{2}{|l|}{ Sector of secondary hospital } \\
\hline \multirow{2}{*}{\multicolumn{2}{|c|}{$\begin{array}{l}\text { Primary length of stay† longer } \\
\text { than cohort median }\end{array}$}} & Community (ref) & 1.00 \\
\hline & & Academic & $0.70(0.59-0.82)$ \\
\hline No (ref) & 1.00 & \multicolumn{2}{|l|}{ Case volume of secondary hospital† } \\
\hline Yes & $1.20(1.17-1.23)$ & Lower (ref) & 1.00 \\
\hline \multicolumn{2}{|l|}{$\begin{array}{l}\text { Hospital-free interval† longer } \\
\text { than cohort median }\end{array}$} & \multirow[t]{3}{*}{ Higher } & $1.04(0.86-1.25)$ \\
\hline No (ref) & 1.00 & & \\
\hline Yes & $1.03(1.00-1.05)$ & & \\
\hline \multicolumn{4}{|c|}{$\begin{array}{l}\text { Note: } \mathrm{Cl}=\text { confidence interval, } \mathrm{OR}=\text { odds ratio, ref = reference group. } \\
\text { `Adjusted for age, sex, neighbourhood average household income, rural residence, year of readmission, Charlson Comorbidity Index, primary length of stay, most } \\
\text { responsible diagnosis at readmission to hospital, arrival by ambulance at readmission, residence in chronic care facility at readmission, admission to hospital in previous } \\
\text { year, physician clinic visits in previous year, hospital-free interval, case volume of secondary hospital and sector of secondary hospital. } \\
\text { †Analyzed as continuous data but presented here in categories to facilitate interpretation. } \\
\text { †Missing data were coded as such and included in analyses; however, missing data were uncommon }(<0.003 \% \text { of all data) and are not presented here. } \\
\text { §The } 5 \text { diagnostic categories with the greatest effect on mortality and the } 5 \text { with the least effect are presented here. For the complete list, see eTable } 8 \text { in Appendix } 1 \text {. }\end{array}$} \\
\hline
\end{tabular}


the adjusted odds of death at 90, 180 or 365 days after readmission were no different for original- and alternative-hospital readmissions (e'Table 11 in Appendix 1).

\section{Sensitivity analyses}

Retaining only the first rather than the most recent readmission for each patient resulted in a similar summary effect estimate for the primary outcome: 20571 (12.7\%) of the 161955 patients readmitted to the original hospital died, as compared with $5780(16.0 \%)$ of the 36194 patients readmitted to an alternative hospital died (unadjusted OR 1.30, 95\% CI 1.271.35; adjusted OR 1.08, 95\% CI 1.04-1.12). Analyzing by primary rather than secondary hospital had little effect on the summary effect measure generated by the multivariable GEE model (adjusted OR 1.08, 95\% CI 1.01-1.16), despite more pronounced influences on the unadjusted hospital-specific effect estimates (eFigure 4 in Appendix 1). Extending the definition of hospital-to-hospital transfer to exclude patients

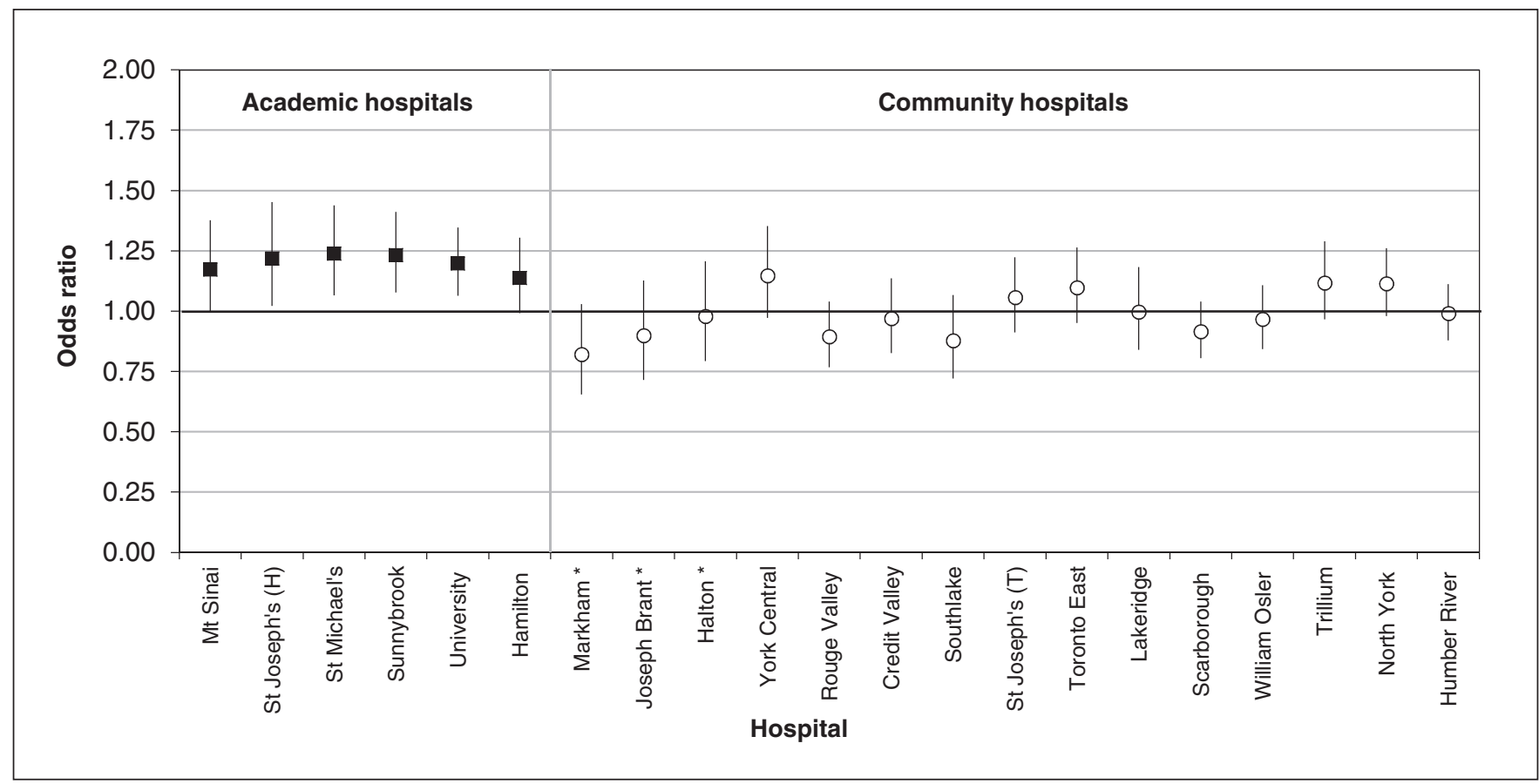

Figure 2: Hospital-specific adjusted odds of death within 30 days after readmission among patients readmitted to an alternative hospital compared with those readmitted to the original hospital. Values greater than 1.0 indicate an increased risk of death. Error bars represent $95 \%$ confidence intervals. *Multivariable logistic regression produced unstable estimates due to nonconvergence.

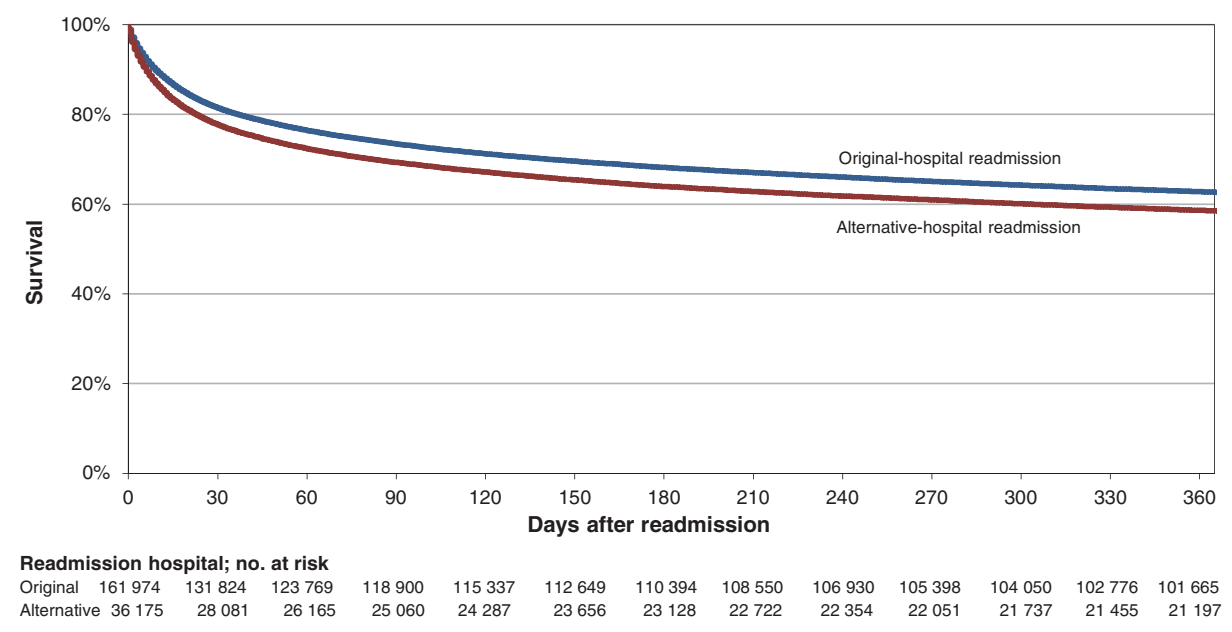

Figure 3: Difference in unadjusted Kaplan-Meier survival curves for patients following readmission to the original hospital and an alternative hospital $(p<0.001)$. 
readmitted within 48 hours after discharge from the primary hospital resulted in a smaller cohort $(n=187446)$, but did not influence the primary outcome summary effect measure (adjusted OR 1.05, 95\% CI 1.02-1.09).

\section{Interpretation}

In this population-based observational cohort study, readmission to an alternative hospital rather than the original hospital was associated with increased patient mortality that was attenuated substantially after adjustment for patient- and hospitallevel covariables. One interpretation of these findings is that alternative-hospital readmission can compromise patient safety, in accord with past studies examining discontinuous care. ${ }^{46-48}$ Another interpretation is that increased patient mortality reflects residual confounding by unmeasured covariables that differed for original- and alternative-hospital patients in our cohort. The latter interpretation acknowledges that conditions with relatively poor prognoses might also predispose patients to alternative-hospital readmission and that system factors promoting alternative-hospital readmission (e.g., ambulance diversion or prolonged wait times in the emergency department) potentially delay care and adversely affect patient outcomes. ${ }^{49,50}$ Given the potential importance of our findings in terms of patient outcomes, further examination of the underlying association between alternative-hospital readmission and mortality is warranted.

Our exploratory analyses found an association between alternative-hospital readmission and mortality for secondary hospitals in the academic sector, but not for those in the community sector. Exploratory comparisons of specific hospital sector transitions also suggested that sector influences the association between readmission to an alternative hospital and mortality (eTable 9 in Appendix 1). One explanation for this finding is that academic hospitals care for highly complex patients who might be particularly vulnerable to discontinuities in care. ${ }^{31} \mathrm{~A}$ second explanation is the confounding that might be introduced if patients with severe or unusual illnesses were directed to subspecialty academic centres for readmission. A third explanation focuses on differences in organizational culture between academic and community hospitals. ${ }^{50}$ Further research, including organizational network analysis, may help clarify this finding. ${ }^{51,52}$

Several features distinguish our study from past research. ${ }^{11}$ We defined alternative-hospital versus original-hospital readmission in advance as the primary predictor of interest. Our patient cohort was population-based, universally insured, selected over an extended interval and substantial in size. We focused on a large contiguous metropolitan area and excluded rural hospitals with potentially idiosyncratic readmission patterns. Each hospital in our study contributed a large number of patients (average of 9455 per hospital), which allowed analyses to account for actual readmission site. Finally, a high proportion of our cohort died within 30 days after readmission, which suggests that our patients had a substantial burden of comorbidity, a high acuity of presenting illness or some combination of these factors.

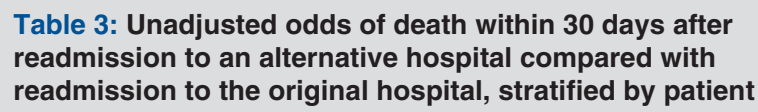

Patient characteristic

Unadjusted $\mathrm{OR}^{\star}(95 \% \mathrm{Cl})$

Age, yr

$<65$

$1.36(1.27-1.46)$

$65-74$

$1.22(1.11-1.33)$

$75-84$

$1.16(1.09-1.23)$

$\geq 85$ $1.15(1.07-1.23)$

Sex

Female $1.35(1.26-1.45)$

Male $1.16(1.08-1.25)$

Neighbourhood household income

Highest quintile $1.27(1.14-1.42)$

Next to highest quintile $\quad 1.22(1.11-1.33)$

Middle quintile $1.23(1.13-1.34)$

Next to lowest quintile $\quad 1.25(1.17-1.34)$

Lowest quintile $1.28(1.19-1.37)$

Charlson Comorbidity Index

$\begin{array}{ll}\leq 1 & 1.42(1.24-1.63) \\ \geq 2 & 1.17(1.10-1.24)\end{array}$

Admissions to hospital in previous year

$1 \quad 1.46(1.35-1.58)$

$\geq 2 \quad 1.09(1.01-1.18)$

Physician clinic visits in previous year

$\leq 6 \quad 1.32(1.21-1.43)$

$\geq 7 \quad 1.23(1.15-1.32)$

Most responsible diagnosis for readmission to hospital†

\begin{tabular}{ll}
\hline Injury & $2.27(1.72-2.99)$ \\
\hline Diverticular disease & $2.25(1.43-3.55)$ \\
\hline Intestinal obstruction & $1.80(1.32-2.45)$ \\
\hline Abdominal pain & $1.61(0.61-4.23)$ \\
\hline Skin infection & $1.53(1.00-2.35)$ \\
\hline Acute coronary syndrome & $0.93(0.80-1.07)$ \\
\hline Venous thromboembolism & $0.89(0.67-1.19)$ \\
\hline Chest pain not otherwise specified & $0.75(0.29-1.90)$ \\
\hline Syncope and collapse & $0.64(0.25-1.63)$ \\
\hline Chronic ischemic heart disease & $0.52(0.35-0.76)$ \\
\hline Primary length of stay longer than cohort median & \\
\hline No & $1.26(1.16-1.36)$ \\
\hline Yes & $1.25(1.15-1.35)$ \\
\hline Hospital-free interval longer than cohort median & \\
\hline No & $1.30(1.19-1.42)$ \\
\hline Yes & $1.20(1.13-1.27)$ \\
\hline Residence in chronic care facility at readmission & \\
\hline No & $1.25(1.17-1.33)$ \\
\hline Yes & $0.99(0.89-1.11)$ \\
\hline Arrival by ambulance at readmission & \\
\hline No & $1.19(1.07-1.31)$ \\
\hline Yes & $1.02(0.96-1.08)$ \\
\hline $\begin{array}{l}\text { Note: Cl }=\text { confidence interval, OR = odds ratio. } \\
{ }^{*} \text { Calculated for each stratum using unadjusted univariable general estimating } \\
\text { equations models. } \\
\text { the } 5 \text { diagnostic categories with the greatest effect on the unadjusted risk of } \\
\text { death within } 30 \text { days after readmission to an alternative hospital and the } 5 \text { with } \\
\text { the least effect are presented here. For the complete list of unadjusted results } \\
\text { stratified by diagnosis at secondary admission, see eTable } 10 \text { in Appendix 1. }\end{array}$ \\
\hline
\end{tabular}




\section{Limitations}

Our study has several limitations inherent in an observational design. Detailed information, such as clinical history, findings on physical examination, laboratory data, patient preferences, cause of death and indicators of hospital performance, were not available. We had no information on the factors motivating alternative-hospital readmission (e.g., dissatisfaction with prior care, geographic proximity when illness recurred, specialist availability) and could not assess the modifiability of readmission patterns. Information about features that might independently influence a patient's emergency department selection and their health outcomes (e.g., homelessness, frequency of motor vehicle travel and awareness of institutional expertise) was not available. Our focus on a single region also limits the generalizability of the results. The mechanism by which alternative-hospital readmission might increase the risk of death remains a matter of conjecture.

\section{Conclusion}

Among patients readmitted within 30 days after discharge, readmission to an alternative hospital was associated with a higher risk of death than readmission to the original hospital. Our study has several implications for patients, clinicians and policy-makers. Before discharge, patients might be informed of the potential advantages of returning to their original hospital should they relapse and require emergency care. Primary care clinicians and prehospital medical service providers might wish to inquire about recent admissions and direct patients back to their original hospital where feasible. Health care financing policies that pay hospitals partly based on observed readmission rates should consider the large numbers of patients readmitted to alternative hospitals and the distinctly high burden of illness associated with such readmissions. In the interim, the data suggest that readmissions to alternative hospitals are worrisome. Whether this adverse prognosis reflects a true causal relation or residual confounding requires further investigation.

\section{References}

1. Jencks SF. Defragmenting care. Ann Intern Med 2010;153:757-8.

2. All-cause readmission to acute care and return to the emergency department. Ottawa (ON): Canadian Institute for Health Information; 2012.

3. Jencks SF, Williams MV, Coleman EA. Rehospitalizations among patients in the Medicare fee-for-service program. N Engl 7 Med 2009;360:1418-28.

4. Allaudeen N, Schnipper JL, Orav EJ, et al. Inability of providers to predict unplanned readmissions. $\mathcal{F}$ Gen Intern Med 2011;26:771-6.

5. Kansagara D, Englander H, Salanitro A, et al. Risk prediction models for hospital readmission: a systematic review. 7AMA 2011;306:1688-98.

6. van Walraven C, Bennett C, Jennings A, et al. Proportion of hospital readmissions deemed avoidable: a systematic review. CMAf 2011;183:E391-402.

7. Hansen LO, Young RS, Hinami K, et al. Interventions to reduce 30-day rehospitalization: a systematic review. Ann Intern Med 2011;155:520-8.

8. Lanièce I, Couturier P, Dramé M, et al. Incidence and main factors associated with early unplanned hospital readmission among French medical inpatients aged 75 years and over admitted through emergency units. Age Ageing 2008;37:416-22.

9. Glynn N, Bennett K, Silke B. Emergency medical readmission: long-term trends and impact on mortality. Clin Med 2011;11:114-8.

10. Nasir K, Lin Z, Bueno H, et al. Is same-hospital readmission rate a good surrogate for all-hospital readmission rate? Med Care 2010;48:477-81.

11. Kind AJ, Bartels C, Mell MW, et al. For-profit hospital status and rehospitalizations at different hospitals: an analysis of Medicare data. Ann Intern Med 2010; 153:718-27.

12. Stiell A, Forster AJ, Stiell IG, et al. Prevalence of information gaps in the emergency department and the effect on patient outcomes. CMA7 2003;169:1023-8.

13. Kripalani S, LeFevre F, Phillips CO, et al. Deficits in communication and information transfer between hospital-based and primary care physicians: implications for patient safety and continuity of care. FAMA 2007;297:831-41.
14. Gaieski DF, Mikkelsen ME, Band RA, et al. Impact of time to antibiotics on survival in patients with severe sepsis or septic shock in whom early goal-directed therapy was initiated in the emergency department. Crit Care Med 2010; 38:1045-53.

15. Goldberg RJ, Mooradd M, Gurwitz JH, et al. Impact of time to treatment with tissue plasminogen activator on morbidity and mortality following acute myocardial infarction (The second National Registry of Myocardial Infarction). Am 7 Cardiol 1998;82:259-64.

16. Lees KR, Bluhmki E, von Kummer R, et al. Time to treatment with intravenous alteplase and outcome in stroke: an updated pooled analysis of ECASS, ATLANTIS, NINDS, and EPITHET trials. Lancet 2010;375:1695-703.

17. van Walraven C, Mamdani M, Fang J, et al. Continuity of care and patient outcomes after hospital discharge. 7 Gen Intern Med 2004;19:624-31.

18. Otter JA, Yezli S, French GL. The role played by contaminated surfaces in the transmission of nosocomial pathogens. Infect Control Hosp Epidemiol 2011; 32:687-99.

19. Croskerry P. The importance of cognitive errors in diagnosis and strategies to minimize them. Acad Med 2003;78:775-80.

20. Reason J. Human error: models and management. BMF 2000;320:768-70.

21. Pham JC, Patel R, Millin MG, et al. The effects of ambulance diversion: a comprehensive review. Acad Emerg Med 2006;13:1220-7.

22. Trzeciak S, Rivers EP. Emergency department overcrowding in the United States: an emerging threat to patient safety and public health. Emerg Med $\mathcal{F}$ 2003;20:402-5.

23. Luft HS, Garnick DW, Mark DH, et al. Does quality influence choice of hospital? JAMA 1990;263:2899-906.

24. Kolstad JT, Chernew ME. Quality and consumer decision making in the market for health insurance and health care services. Med Care Res Rev 2009;66(1 Suppl):28S-52S.

25. The big move: transforming transportation in the Greater Toronto and Hamilton Area. Toronto (ON): Greater Toronto Transit Authority; 2008:111.

26. 2001 census: 2001 community profiles. Ottawa (ON): Statistics Canada; 2002 (updated 2007 Apr. 4). Cat no. 93F0053XIE. Available: www12.statcan.ca/english /Profil01/CP01/Index.cfm?Lang=E (accessed 2012 June 25).

27. Canada Health Act, 1984 , c. 6, s. 10.

28. Quan H, Sundararajan V, Halfon P, et al. Coding algorithms for defining comorbidities in ICD-9-CM and ICD-10 administrative data. Med Care 2005; 43:1130-9.

29. Hospital report 2003: acute care. Ottawa (ON): Canadian Institute for Health Information; 2003. Available: https://secure.cihi.ca/free_products/OHA_2003 _e.pdf (accessed 2014 Mar. 11).

30. Allison JJ, Kiefe CI, Weissman NW, et al. Relationship of hospital teaching status with quality of care and mortality for Medicare patients with acute MI. 7AMA 2000;284:1256-62.

31. Shahian DM, Nordberg P, Meyer GS, et al. Contemporary performance of US teaching and nonteaching hospitals. Acad Med 2012;87:701-8.

32. Ross JS, Normand SL, Wang Y, et al. Hospital volume and 30-day mortality for three common medical conditions. N Engl f Med 2010;362:1110-8.

33. Birkmeyer JD, Siewers AE, Finlayson EV, et al. Hospital volume and surgical mortality in the United States. NEngl 7 Med 2002;346:1128-37.

34. Tu JV, Pashos CL, Naylor CD, et al. Use of cardiac procedures and outcomes in elderly patients with myocardial infarction in the United States and Canada. N Engl f Med 1997;336:1500-5.

35. Elixhauser A, Steiner C, Palmer L. Clinical classifications software (CCS) for ICD9-CM. Rockville (MD): Agency for Healthcare Research and Quality; 2012. Available: www.hcup-us.ahrq.gov/toolssoftware/ccs/ccs.jsp (accessed 2012 Aug. 21)

36. Guttmann A, Schull MJ, Vermeulen MJ, et al. Association between waiting times and short term mortality and hospital admission after departure from emergency department: population based cohort study from Ontario, Canada. $B M 72011 ; 342: \mathrm{d} 2983$.

37. Gruneir A, Dhalla IA, van Walraven C, et al. Unplanned readmissions after hospital discharge among patients identified as being at high risk for readmission using a validated predictive algorithm. Open Med 2011;5:e104-11.

38. Williams JI, Young W. A summary of studies on the quality of health care administrative databases in Canada. In: Goel V, Williams JI, Anderson GM, editors. Patterns of health care in Ontario: the ICES practice atlas. $2^{\text {nd }}$ ed. Ottawa (ON): Canadian Medical Association; 1996:339-45.

39. Juurlink DN, Preyra C, Coxford R, et al. Canadian Institute for Health Information discharge abstract database: a validation study. Toronto $(\mathrm{ON})$ : Institute for Clinical Evaluative Sciences; 2006.

40. Iron K, Zagorski BM, Sykora K, et al. Living and dying in Ontario: an opportunity for improved bealth information. ICES investigative report. Toronto (ON): Institute for Clinical Evaluative Sciences; 2008.

41. Krieger N. Overcoming the absence of socioeconomic data in medical records: validation and application of a census-based methodology. Am 7 Public Health 1992;82:703-10.

42. Rajan C, editor. The Canadian bealth facilities directory. 4th ed. North York (ON): Business Information Group; 2002.

43. Jackson T, editor. The guide to Canadian health facilities. Vol. 10. Ottawa (ON): The Canadian Hospital Association Press; 2002.

44. Jackson T, editor. The guide to Canadian health facilities. Vol. 12. Ottawa (ON): The Canadian Hospital Association Press; 2004. 
45. Hubbard AE, Ahern J, Fleischer NL, et al. To GEE of not to GEE: comparing population average and mixed models for estimating associations between neighborhood risk factors and health. Epidemiology 2010;21:467-74.

46. Moore C, Wisnivesky J, Williams S, et al. Medical errors related to discontinuity of care from an inpatient to an outpatient setting. 7 Gen Intern Med 2003; 18:646-51.

47. Kripalani S, LeFevre F, Phillips CO, et al. Deficits in communication and information transfer between hospital-based and primary care physicians: implications for patient safety and continuity of care. FAMA 2007;297:831-41.

48. Hill AB. The environment and disease: Association or causation? Proc R Soc Med 1965;58:295-300

49. Shen YC, Hsia RY. Association between ambulance diversion and survival among patients with acute myocardial infarction. FAMA 2011;305:2440-7.

50. Hartmann CW, Meterko M, Rosen AK, et al. Relationship of hospital organizational culture to patient safety climate in the Veterans Health Administration. Med Care Res Rev 2009;66:320-38.

51. Lee BY, McGlone SM, Song Y, et al. Social network analysis of patient sharing among hospitals in Orange County, California. Am 7 Public Health 2011;101: 707-13.

52. Donker T, Wallinga J, Grundmann H. Patient referral patterns and the spread of hospital-acquired infections through national health care networks. PLoS Comput Biol 2010;6:e1000715.

Affiliations: Institute for Clinical Evaluative Sciences (Staples, Thiruchelvam), Toronto, Ont.; Division of General Internal Medicine (Staples), University of Washington, Seattle, Wash.; Department of Medicine (Redelmeier), University of Toronto; Evaluative Clinical
Sciences Platform (Redelmeier), Sunnybrook Health Sciences Centre, Toronto, Ont.

Contributors: All of the authors contributed equally to the design and conduct of the study. John Staples and Deva Thiruchelvam had full access to all the data in the study and take responsibility for the integrity of the data and the accuracy of the data analysis. All of the authors contributed to the interpretation of the data. John Staples prepared the initial draft of the manuscript. All of the authors revised the manuscript, approved the final version submitted for publication and agreed to act as guarantors of the work.

Funding: The study was funded by the Canadian Institutes of Health Research and by funding associated with Donald Redelmeier's Canada Research Chair in Medical Decision Sciences. Neither funding organization had any role in the design of the study; in the collection, analysis or interpretation of the data; in the preparation, review or approval of the manuscript; or in the decision to submit the manuscript for publication.

Acknowledgement: The authors thank William Chan, Mara Hansen, Finlay McAlister, Kelley Ross, Michael Schull, Michael Schwandt, Therese Stukel and Carl van Walraven for their insightful comments.

Supplemental information: For reviewer comments and the original submission of this manuscript, please see www.cmajopen.ca/content/2/2 /E77/suppl/DC1 